\title{
Enhanced Radar Imaging in Uncertain Environment: A Descriptive Experiment Design Regularization Approach
}

\author{
Yuriy Shkvarko, ${ }^{1}$ Hector Perez-Meana, ${ }^{1}$ and Alejandro Castillo-Atoche ${ }^{2}$ \\ ${ }^{1}$ ESIME, Unidad Culhuacan, Avenida Santa Ana no. 1000, Colonia San Francisco Culhuacan, México D.F., C.P. 04430, Mexico \\ ${ }^{2}$ CINVESTAV, Unidad Guadalajara, Avenida Científica no. 1145, Colonia El Bajío, Zapopan, Jalisco, C.P. 45015, Mexico
}

Correspondence should be addressed to Alejandro Castillo-Atoche, acastill@uady.mx

Received 4 February 2008; Accepted 29 May 2008

Recommended by M. Greco

A new robust technique for high-resolution reconstructive imaging is developed as required for enhanced remote sensing (RS) with imaging array radar or/and synthetic aperture radar (SAR) operating in an uncertain RS environment. The operational scenario uncertainties are associated with the unknown statistics of perturbations of the signal formation operator (SFO) in turbulent medium, imperfect array calibration, finite dimensionality of measurements, uncontrolled antenna vibrations, and random carrier trajectory deviations in the case of SAR. We propose new descriptive experiment design regularization (DEDR) approach to treat the uncertain radar image enhancement/reconstruction problems. The proposed DEDR incorporates into the minimum risk (MR) nonparametric estimation strategy the experiment design-motivated operational constraints algorithmically coupled with the worst-case statistical performance (WCSP) optimization-based regularization. The MR objective functional is constrained by the WCSP information, and the robust DEDR image reconstruction operator applicable to the scenarios with the low-rank uncertain estimated data correlation matrices is found. We report and discuss some simulation results related to enhancement of the uncertain SAR imagery indicative of the significantly increased performance efficiency gained with the developed approach.

Copyright (c) 2008 Yuriy Shkvarko et al. This is an open access article distributed under the Creative Commons Attribution License, which permits unrestricted use, distribution, and reproduction in any medium, provided the original work is properly cited.

\section{INTRODUCTION}

Modern applied theory of reconstructive radar imaging is now a mature and well-developed research field, presented and detailed in many works (see, e.g., $[1,2]$ and references therein). The classical imaging with array radar or SAR implies application of a method called "matched spatial filtering" to process the recorded data signals $[1,3,4]$. Stated formally [1], such a method implies application of the adjoint signal formation operator (SFO) to the recorded data, squared detection of the filter outputs, and their averaging over the actually recorded samples (the socalled snapshots [5]) of the independent data observations. Although a number of authors have proposed different linear and nonlinear postprocessing approaches to enhance the images formed using such matched estimator (see, e.g., [3, 5$8]$ ), all those are not a direct inference from the Bayesian statistically optimal estimation theory [4]. Other approaches had focused primarily on designing the constrained regularization techniques for improving the resolution of the closely spaced components in the power spatial spectrum pattern (SSP) obtained by ways different from the matched spatial filtering [9-12], but again without aggregating the regularization principles with the minimum risk estimation strategy. Although the existing theory offers a manifold of statistical and descriptive regularization techniques for reconstructive imaging, in many application areas there still remain some unresolved crucial theoretical and processing problems related to large scale sensor array radar/SAR reconstructive imaging in uncertain operational scenarios.

The predominant challenge of this study is to solve the SSP reconstruction problem in context of the uncertain environment. Thus, the problem of enhanced imaging of the extended large-scale scenes remotely sensed with an array radar/SAR operating in the uncertain remote sensing (RS) environment is stated and treated as an ill-conditioned statistical nonlinear inverse problem. The operational uncertainties are associated with the unknown statistics of perturbations of the SFO in the turbulent medium, imperfect array calibration, finite dimensionality of measurements, uncontrolled antenna vibrations, and random carrier trajectory deviations in the case of SAR. New descriptive experiment 
design regularization (DEDR) approach to radar imaging in the uncertain environment is addressed to perform the enhanced reconstruction of the power spatial spectrum pattern (SSP) of the scattered wavefield from the uncertain data measurements. The proposed DEDR incorporates into the minimum risk (MR) nonparametric estimation strategy the DEDR-motivated constraints of the observability of the initial scene scattering wavefield algorithmically coupled with the worst-case statistical performance (WCSP) optimizationbased regularization. The MR objective function is constrained by the WCSP information, and the DEDR technique for robust image reconstruction applicable to the scenarios with the low-rank uncertain estimated data correlation matrices is found. Pursuing such an approach, we establish a family of the robust DEDR-related estimators that encompass a manifold of the imaging techniques ranging from traditional array matched spatial filtering to new DEDRrelated robust adaptive array beamforming. We also present the robust DEDR-related imaging algorithms that manifest enhanced resolution of the reconstructed array images with substantially decreased computational load. The efficiency of two general DEDR-related algorithms (the robust spatial filtering (RSF) algorithm and the robust adaptive spatial filtering (RASF) algorithm) is illustrated through computer simulations of reconstructing the digital images provided with the SAR operating in some typical uncertain remote sensing scenarios.

\section{DESCRIPTIVE EXPERIMENT DESIGN REGULARIZATION FORMALISM}

\subsection{Problem model}

Consider a coherent RS experiment in a random medium and the narrowband assumption $[1,4,6]$ that enables us to model the extended object backscattered field by imposing its time invariant complex scattering (backscattering) function $e(\mathbf{x})$ in the scene domain (scattering surface) $X \ni \mathbf{x}$. The measurement data wavefield $u(\mathbf{y})=s(\mathbf{y})+n(\mathbf{y})$ consists of the echo signals $s$ and additive noise $n$ and is available for observations and recordings within the prescribed timespace observation domain $Y=T \times P$, where $\mathbf{y}=(t, \mathbf{p})^{\mathrm{T}}$ defines the time-space points in $Y$. The model of the observation wavefield $u$ is defined by specifying the stochastic equation of observation (EO) of an operator form $[1,13]$ : $u=\widetilde{S} e+n ; e \in \mathrm{E} ; u, n \in \mathrm{U} ; \widetilde{S}: \mathrm{E} \rightarrow \mathrm{U}$, in the Hilbert signal spaces $\mathrm{E}$ and $\mathrm{U}$ with the metrics structures induced by the inner products, $\left[u_{1}, u_{2}\right]_{\mathrm{U}}=\int_{Y} u_{1}(\mathbf{y}) u_{2}^{*}(\mathbf{y}) d \mathbf{y}$ and $\left[e_{1}, e_{2}\right]_{\mathrm{E}}=\int_{X} e_{1}(\mathbf{x}) e_{2}^{*}(\mathbf{x}) d \mathbf{x}$, respectively. The operator model of the stochastic EO in the conventional integral form $[1,13]$ may be written as

$$
u(\mathbf{y})=(\widetilde{S} e(\mathbf{x}))(\mathbf{y})+n(\mathbf{y})=\int_{X} \tilde{S}(\mathbf{y}, \mathbf{x}) e(\mathbf{x}) d \mathbf{x}+n(\mathbf{y})
$$

The random functional kernel $\widetilde{S}(\mathbf{y}, \mathbf{x})$ of the stochastic integral SFO $\widetilde{S}$ given by (1) defines the signal wavefield formation model. Its mean, $S(\mathbf{y}, \mathbf{x})=\langle\widetilde{S}(\mathbf{y}, \mathbf{x})\rangle$, is referred to as the nominal SFO in the RS measurement channel specified by the time-space modulation of signals employed in a particular radar system $[3,8]$ and the variations about the mean $\delta S(\mathbf{y}, \mathbf{x})=\widetilde{S}(\mathbf{y}, \mathbf{x})-S(\mathbf{y}, \mathbf{x})$ model the model uncertainties and random perturbations of the wavefield at different propagation paths (the so-called extended Rytov's model [1]).

We assume an incoherent nature of the backscattered field $e(\mathbf{x})$. This is naturally inherent to the RS experiments and leads to the $\delta$-form of the object field correlation function, $R_{e}\left(\mathbf{x}, \mathbf{x}^{\prime}\right)=b(\mathbf{x}) \delta\left(\mathbf{x}-\mathbf{x}^{\prime}\right)$, where $e(\mathbf{x})$ and $b(\mathbf{x})=$ $\left\langle|e(\mathbf{x})|^{2}\right\rangle$ are referred to as the scene random complex scattering function and its average power scattering function or spatial spectrum pattern (SSP), respectively. The radar imaging problem is to derive an estimate $\hat{b}(\mathbf{x})$ of the SSP

$$
b(\mathbf{x})=(\mathscr{B} e)(\mathbf{x})=\operatorname{Aver}^{(2)}\{e(\mathbf{x})\}=\left\langle e(\mathbf{x}) e^{*}(\mathbf{x})\right\rangle
$$

(referred to as the desired RS image) by processing the available finite dimensional array radar/SAR measurements of the data wavefield $u(\mathbf{y})$, where $\mathcal{B}$ defines the second-order statistical averaging operator.

\subsection{Projection formalism for data representation}

Viewing it as an approximation problem leads one to a projection concept for a reduction of the data field $u(\mathbf{y})$ to the $M$-D spatial-temporal data recordings:

$$
\mathbf{u}=\underset{m}{\operatorname{vec}}\left\{u_{m}=\left[u, h_{m}\right]_{U} ; m=1, \ldots, M\right\}
$$

composed of the expansion/decomposition coefficients $\left\{u_{m}=\left[u, h_{m}\right]_{U} ; m=1, \ldots, M\right\}$, where $\left\{h_{m}(\mathbf{y})\right\}$ defines the set of orthogonal normalized basis functions in the $M$ $D$ data approximation subspace $\mathrm{U}_{(M)}=P_{\mathrm{U}(M)} \mathrm{U}$ [13]. These are defined via corresponding compositions of the calibrated antenna array tapering functions and sampling filters that explicitly specify the corresponding data projection operator $P_{\mathrm{U}(M)}$ (see [13-15] for details).

In analogy to (3), one can define now the $K$-D vectorform approximation of the scene random scattering function as follows:

$$
\mathbf{e}=\underset{k}{\operatorname{vec}}\left\{e_{k}=\left[e, g_{k}\right]_{\mathrm{E}} ; k=1, \ldots, K\right\} .
$$

The elements of vector (4) are composed of the decomposition coefficients $\left\{e_{k}=\left[e, g_{k}\right]_{\mathrm{E}} ; k=1, \ldots, K\right\}$ with respect to some chosen normalized orthogonal set of expansion functions $\left\{g_{k}(\mathbf{x})\right\}$ that span such $K-\mathrm{D}$ signal approximation subspace $\mathrm{E}_{(K)}=P_{\mathrm{E}(K)} \mathrm{E}$ and specify the corresponding scene wavefield projection operator $P_{\mathrm{E}(K)}$.

The descriptive experiment design (DED) aspects of the SSP reconstruction problem involving the analysis of how to choose the basis functions $\left\{g_{k}(\mathbf{x})\right\}$ that span the signal representation subspace $\mathrm{E}_{(K)}=P_{\mathrm{E}(K)} \mathrm{E}=\operatorname{Span}\left\{g_{k}\right\}$ for a given observation subspace $\mathrm{U}_{(M)}=\operatorname{Span}\left\{h_{m}\right\}$ were investigated in more details in the previous studies [13, 15]. Following [15], in the rest of this study, we consider the conventional (i.e., ordinary rectangular pixel format) representation basis over a $K_{x 2} \times K_{x 1}$ regular pixel-formatted lattice $[14,16]$, 
where $K_{x 1}$ defines the dimension of the rectangular grid over the horizontal (azimuth) coordinate $x_{1}$, and $K_{x 2}$ defines its dimension over the orthogonal (range) coordinate $x_{2}$ (the number of the slant range gates projected onto the scene frame). Such regular lattice of points is next specified by the ordered multi-index $k=\left(k_{x 1}, k_{x 2}\right) ; k_{x 1}=1, \ldots, K_{x 1}$; $k_{x 2}=1, \ldots, K_{x 2} ; k=1, \ldots, K=K_{x 1} \times K_{x 2}$.

\subsection{Uncertain finite-dimensional observations}

In the DED formalism, an imperfect calibration of the array (due to displacements of some array elements with respect to the presumed nominal positions, as well as distorted antennas shapes $[4,9])$ is attributed to the unknown disturbances $\left\{\delta h_{m} ; m=1, \ldots, M\right\}$ in the decomposition functions $\left\{\tilde{h}_{m}=h_{m}+\delta h_{m}\right\}$ in (3). In imaging SAR applications, such disturbances incorporate the deviations of a carrier from the nominal trajectory and antenna vibration $[3,17]$. These disturbances and propagation perturbations result in the uncertain SFO matrix:

$$
\widetilde{\mathbf{S}}=\mathbf{S}+\Delta .
$$

In (5), the nominal $M \times K$ SFO matrix $\mathrm{S}$ is composed of the elements $\left\{S_{m k}=\left[S g_{k}, h_{m}\right]_{\mathrm{U}}\right\}$, while all problem model uncertainties are attributed to the distortion term, in which the elements of the uncertainty matrix $\Delta$ are treated as unknown values (realizations of random variables) with an unknown probability density function (pdf) $p(\Delta)$.

\subsection{Vector-form equation of observation}

Now, we proceed from the stochastic integral-form EO (1) to its finite-dimensional approximation (vector) form:

$$
\mathbf{u}=\widetilde{\mathbf{S e}}+\mathbf{n}=\mathbf{S e}+\Delta \mathbf{e}+\mathbf{n},
$$

in which the disturbed SFO matrix is defined by (5), and $\mathbf{e}, \mathbf{n}, \mathbf{u}$ represent zero-mean vectors composed of the decomposition coefficients $e_{k}, n_{m}$, and $u_{m}$, respectively. These vectors are characterized by the correlation matrices: $\mathbf{R}_{\mathbf{e}}=\mathbf{D}=\mathbf{D}(\mathbf{b})=\operatorname{diag}\{\mathbf{b}\}$ (a diagonal matrix with vector b at its principal diagonal), $\mathbf{R}_{\mathbf{n}}$, and $\mathbf{R}_{\mathbf{u}}=\left\langle\widetilde{\mathbf{S}}_{\mathbf{e}} \widetilde{\mathbf{S}}^{+}\right\rangle_{p(\Delta)}+\mathbf{R}_{\mathbf{n}}$, respectively, where $\langle\cdot\rangle_{p(\Delta)}$ defines the averaging performed over the randomness of $\Delta$ characterized by the unknown probability density function $p(\boldsymbol{\Delta})$. Vector $\mathbf{b}$ is composed of the elements, $b_{k}=\mathscr{B}\left(e_{k}\right)=\left\langle e_{k} e_{k}^{*}\right\rangle=\left\langle\left|e_{k}\right|^{2}\right\rangle ; k=1, \ldots, K$ and is referred to as a $K-\mathrm{D}$ vector-form representation of the SSP.

We refer to the estimate, $\hat{\mathbf{b}}$, as a discrete-form representation of the desired SSP, that is, the brightness image of the wavefield sources distributed over the pixel-formatted object scene remotely sensed with an employed array radar/SAR. Thus, the uncertain SSP reconstruction problem can be reformulated now as follows: to derive an estimator for reconstructing the $K-\mathrm{D}$ approximation:

$$
\hat{b}_{(K)}(\mathbf{x})=\sum_{k=1}^{K} \hat{b}_{k}\left|g_{k}(\mathbf{x})\right|^{2}=\mathbf{g}^{T}(\mathbf{x}) \operatorname{diag}\{\hat{\mathbf{b}}\} \mathbf{g}(\mathbf{x})
$$

of the SSP distribution in the environment $X \ni \mathbf{x}$. Note that in applications, we employ the ordinary pixel expansion format [16], while all theoretical results are valid also for any feasible decomposition function basis, $\mathbf{g}(\mathbf{x})=\operatorname{vec}_{k}\left\{g_{k}(\mathbf{x})\right\}$, in (7).

\section{DEDR STRATEGY}

\subsection{Formulation of DEDR estimation strategy}

In the descriptive statistical formalism, the desired SSP vector $\widehat{\mathbf{b}}$ is recognized to be a vector of the principal diagonal of an estimate of the correlation matrix $\mathbf{R}_{\mathbf{e}}(\mathbf{b})$, that is, $\hat{\mathbf{b}}=\left\{\hat{\mathbf{R}}_{\mathbf{e}}\right\}_{\text {diag }}$. Thus, one can seek to estimate the desired SSP $\hat{b}_{(K)}(\mathbf{x})=$ $\mathbf{g}^{T}(\mathbf{x}) \operatorname{diag}\{\hat{\mathbf{b}}\} \mathbf{g}(\mathbf{x})$ given the data correlation matrix $\mathbf{R}_{\mathbf{u}}$ preestimated via averaging of some $J$ independent sampled correlations [6]:

$$
\mathbf{Y}=\widehat{\mathbf{R}}_{\mathbf{u}}=\underset{j \in J}{\operatorname{aver}}\left\{\mathbf{u}_{(j)} \mathbf{u}_{(j)}^{+}\right\}=\frac{1}{J} \sum_{j=1}^{J} \mathbf{u}_{(j)} \mathbf{u}_{(j)}^{+},
$$

and determining the solution operator $(\mathrm{SO})$ F such that

$$
\hat{b}_{(K)}(\mathbf{x})=\mathbf{g}^{T}(\mathbf{x}) \operatorname{diag}\left\{\left\{\mathbf{F Y F}^{+}\right\}_{\text {diag }}\right\} \mathbf{g}(\mathbf{x}) .
$$

To optimize the search for the desired SO F, we formulate here the following DEDR strategy:

$$
\begin{gathered}
\mathbf{F}=\arg \min _{\mathbf{F}}\{\mathfrak{R}(\mathbf{F})\} \\
\text { subject to }\left\langle\|\Delta\|^{2}\right\rangle_{p(\Delta)} \leq \delta,
\end{gathered}
$$

where the conditioning term represents the worst-case statistical performance (WCSP) regularizing constraint imposed on the unknown second-order statistics $\left\langle\|\Delta\|^{2}\right\rangle_{p(\Delta)}$ of the random distortion component $\Delta$ of the SFO matrix (5), and the DEDR "generalized risk" function is defined as

$$
\mathfrak{R}(\mathbf{F})=\operatorname{tr}\left\{\left\langle(\mathbf{F} \tilde{\mathbf{S}}-\mathbf{I}) \mathbf{A}(\mathbf{F} \tilde{\mathbf{S}}-\mathbf{I})^{+}\right\rangle_{p(\Delta)}\right\}+\alpha \operatorname{tr}\left\{\mathbf{F R}_{\mathbf{n}} \mathbf{F}^{+}\right\},
$$

where superscript + defines conjugate transpose. The DEDR strategy (10), (11) implies the minimization of the $\alpha$ weighted sum of the systematic error measure (specified by the first term in the risk function (12)) and noise error (specified by the second term in the risk function (12)) in the desired RSS estimate (9), in which the unknown disturbances of the SFO are treated through the WCSP bounding constraint (11) imposed onto the averaged squared norm of $\Delta$. The selections (adjustments) of the regularization parameter $\alpha$ and the diagonal-form weight matrix $\mathbf{A}$ (the socalled metrics inducing matrix $[13,16])$ with the diagonal composed of positive numbers $\left\{a_{k k}>0 ; k=1, \ldots, K\right\}$ provide the additional DEDR “degrees of freedom" assigning the weights $a_{k k}$ to the particular SSP vector components $b_{k}$. These weights $\left\{a_{k k}\right\}$ are the user-defined parameters that may incorporate any descriptive metrics properties of a solution $[7,8,16]$. In the simplest case of no preference 
to reconstruction of particular SSP components over the observation scene frame, the uniform metrics is typically induced by setting $\mathbf{A}=\mathbf{I}$, that is, the identity matrix. In Section 3.2, we will consider the adaptive DEDR case and specify the corresponding solution-dependent A. Nevertheless, independent on any feasible choice of $\alpha, \mathbf{A}$ in the risk function (12), the conditional optimization problem (10), (11) can be reformulated as

$$
\mathbf{F}=\arg \min _{\mathbf{F}} \max _{\left\langle\|\Delta\|^{2}\right\rangle_{p(\Delta)} \leq \delta}\{\Re(\mathbf{F})\}
$$

\subsection{Decomposition of DEDR risk}

To proceed with the derivation of the estimator (9), (13), we now decompose the risk (12) incorporating directly the WCSP uncertainty constraint into the DEDR strategy. The first term in the risk function (12) specifies the systematic error component as it measures "how far" the desired SO $\mathbf{F}$ is from the pseudoinverse of $\widetilde{\mathbf{S}}$ in the averaged operator metrics. We next, decompose this term into the following:

$$
\operatorname{tr}\left\{\left\langle(\mathbf{F} \tilde{\mathbf{S}}-\mathbf{I}) \mathbf{A}(\mathbf{F} \tilde{\mathbf{S}}-\mathbf{I})^{+}\right\rangle_{p(\Delta)}\right\}=\|\mathbf{F S}-\mathbf{I}\|_{\mathbf{A}}^{2}+\left\langle\|\mathbf{F} \Delta\|_{\mathbf{A}}^{2}\right\rangle_{p(\Delta)},
$$

where $\|\mathbf{C}\|_{\mathbf{A}}^{2}=\operatorname{tr}\left\{\mathbf{C A C}^{+}\right\}$denotes the $\mathbf{A}$-weighted squared operator norm of a matrix, C. The second term in (14) has the statistical meaning of the average noise energy in the resulting solution (9); hence it specifies the fluctuation error measure. This term can be bounded applying the Loewner ordering [16] of the weight matrix $\mathbf{A} \leq y \mathbf{I}$ with the Loewner ordering factor $\gamma=\min \{\bar{\gamma}: \mathbf{A} \leq \bar{\gamma} \mathbf{I}\}>0$ that yields

$$
\left\langle\|\mathbf{F} \Delta\|_{\mathbf{A}}^{2}\right\rangle \leq \gamma\left\langle\|\mathbf{F} \Delta\|^{2}\right\rangle \leq \gamma\|\mathbf{F}\|^{2}\left\langle\|\boldsymbol{\Delta}\|^{2}\right\rangle,
$$

where the second inequality follows from the CauchySchwarz inequality [16], and $\|\mathbf{C}\|^{2}=\|\mathbf{C}\|_{\mathrm{I}}^{2}=\operatorname{tr}\left\{\mathbf{C C}^{+}\right\}$defines a conventional squared norm of a matrix, $C$. Using the constraint (11), we next evaluate the maximum value that may take the last term in the inequality (15), that is,

$$
\max _{\left\langle\|\Delta\|^{2}\right\rangle_{p(\Delta)} \leq \delta}\left\{\gamma\|\mathbf{F}\|^{2}\left\langle\|\Delta\|^{2}\right\rangle_{p(\Delta)}\right\}=\varepsilon\|\mathbf{F}\|^{2}
$$

valid for any given bounding factor $\varepsilon=\delta \gamma \geq 0$. With this evaluation (16), the WCSP-constrained DEDR strategy (13) is transformed into the following nonconstrained optimization problem:

$$
\mathbf{F}=\arg \min _{\mathbf{F}}\{\mathfrak{R} \operatorname{DEDR}(\mathbf{F})\}
$$

with the aggregated DEDR risk functional:

$$
\left\{\Re_{\mathrm{DEDR}}(\mathbf{F})\right\}=\operatorname{tr}\left\{(\mathbf{F S}-\mathbf{I}) \mathbf{A}(\mathbf{F S}-\mathbf{I})^{+}\right\}+\alpha \operatorname{tr}\left\{\mathbf{F R}_{\Sigma} \mathbf{F}^{+}\right\},
$$

where

$$
\mathbf{R}_{\Sigma}=\mathbf{R}_{\Sigma}(\beta)=\left(\mathbf{R}_{\mathbf{n}}+\beta \mathbf{I}\right) ; \quad \beta=\frac{\varepsilon}{\alpha} \geq 0 .
$$

\section{DEDR ESTIMATORS OF SSP}

\subsection{General-form SSP estimator}

Routinely solving the minimization problem (17), we obtain the desired DEDR-optimal SO:

$$
\mathbf{F}_{\mathrm{DEDR}}=\mathbf{K}_{\mathrm{A}, \alpha, \beta} \mathbf{S}^{+} \mathbf{R}_{\Sigma}^{-1}
$$

with the self-adjoint robust reconstruction operator:

$$
\mathbf{K}_{\mathbf{A}, \alpha, \beta}=\left(\mathbf{S}^{+} \mathbf{R}_{\Sigma}^{-1}(\beta) \mathbf{S}+\alpha \mathbf{A}^{-1}\right)^{-1}
$$

dependent on three degrees of freedom: $\alpha, \beta$, and $\mathbf{A}$.

Note, that the derived robust SO (20) involves the Hermitian conjugate $\mathbf{S}^{+}$of the regular SFO $\mathbf{S}$ (i.e., it satisfies the DED-observability requirements [15]) and does not involve the inversion of $\mathbf{Y}$ (i.e., it is applicable to the reconstructive SAR imaging problems with only one-recorded realization of the trajectory data signal available for further processing, $J=1$ ).

The general-form DEDR-optimal SO (20) enables us now to derive the corresponding general-form robust SSP estimator putting (20) into (9) that yields

$$
\begin{aligned}
\hat{b}_{(K)} & (\mathbf{x}) \\
= & \hat{b}_{(K)}(\mathbf{x} \mid \mathbf{A}, \alpha, \beta) \\
= & \mathbf{g}^{T}(\mathbf{x}) \operatorname{diag}\left\{\left\{\mathbf{K}_{\mathbf{A}, \alpha, \beta} \mathbf{S}^{+} \mathbf{R}_{\Sigma}^{-1}(\beta) \mathbf{Y} \mathbf{R}_{\Sigma}^{-1}(\beta) \mathbf{S} \mathbf{K}_{\mathbf{A}, \alpha, \beta}\right\}_{\operatorname{diag}}\right\} \mathbf{g}(\mathbf{x}) .
\end{aligned}
$$

This general-form DEDR estimator for the SSP can also be represented in the alternative form as

$$
\hat{b}_{(K)}(\mathbf{x})=\mathbf{g}^{T}(\mathbf{x}) \operatorname{diag}\left\{\left\{\mathbf{K}_{\mathbf{A}, \alpha, \beta} \underset{j \in J}{\operatorname{aver}}\left\{\mathbf{q}_{(j)} \mathbf{q}_{(j)}^{+}\right\} \mathbf{K}_{\mathbf{A}, \alpha, \beta}\right\}_{\operatorname{diag}}\right\} \mathbf{g}(\mathbf{x}),
$$

where $\mathbf{q}_{(j)}=\mathbf{S}^{+} \mathbf{R}_{\Sigma}^{-1} \mathbf{u}_{(j)}$ is recognized to be an output of the DEDR-regularized matched spatial processing algorithm with noise whitening that assumes the given composed correlation matrix, $\mathbf{R}_{\Sigma}=\mathbf{R}_{\Sigma}(\beta)$. In practical RS scenarios, it is a common practice $[3-5,14]$ to accept the robust white observation noise model, that is, $\mathbf{R}_{\mathbf{n}}^{-1}=\left(1 / N_{0}\right) \mathbf{I}$ and treat the noise intensity $N_{0}$ together with the uncertainty factor $\beta$ in the composed model of $\mathbf{R}_{\Sigma}$ defined by (19).

\subsection{Family of the DEDR-related algorithms}

A family of the DEDR-related algorithms for estimating the SSP can be derived now from (22) via controlling the regularization parameters $\alpha, \beta$, and the weight matrix $\mathbf{A}$ that constitute the degrees of freedom of the developed DEDR method.

\subsubsection{Robust spatial filtering algorithm}

Consider the white zero-mean noise in observations and no preference to any prior model information, that is, putting $\mathbf{A}=\mathbf{I}$. Let the regularization parameter be adjusted as the 
TABLE 1: IOSNR gained with different DEDR-related reconstruction algorithms (results are reported for the first uncertain operational scenario and second scene).

\begin{tabular}{|c|c|c|c|c|}
\hline \multicolumn{5}{|c|}{ Scenario 1: $\Delta \Psi_{a}^{(1)}\left(x_{1}\right)=10 ; \Delta \Psi_{r}\left(x_{2}\right)=3 ; k_{\Delta}=\beta / N_{0}=0.1$} \\
\hline$\mu[\mathrm{dB}]$ & $\begin{array}{l}\text { Nonconstrained RSF } \\
\text { IOSNR }^{(2)}\end{array}$ & $\begin{array}{c}\text { Constrained RSF } \\
\operatorname{IOSNR}^{(3)}\end{array}$ & $\begin{array}{c}\text { Nonconstrained RASF } \\
\operatorname{IOSNR}^{(4)}\end{array}$ & $\begin{array}{c}\text { Constrained RASF (WCSP-optimized) } \\
\operatorname{IOSNR}^{(5)}\end{array}$ \\
\hline 5 & 1.85 & 2.158 & 2.2 & 2.45 \\
\hline 10 & 2.4 & 2.68 & 2.32 & 2.89 \\
\hline 15 & 2.56 & 2.76 & 2.67 & 3.4 \\
\hline 20 & 2.73 & 3.37 & 3.02 & 4.2 \\
\hline 25 & 3.47 & 4.23 & 3.1 & 5.32 \\
\hline 30 & 3.85 & 4.95 & 3.64 & 5.46 \\
\hline
\end{tabular}

TABLE 2: IOSNR gained with different DEDR-related reconstruction algorithms (results are reported for the second uncertain operational scenario and second scene).

\begin{tabular}{|c|c|c|c|c|}
\hline \multicolumn{5}{|c|}{ Scenario $2: \Delta \Psi_{a}^{(1)}\left(x_{1}\right)=14 ; \Delta \Psi_{r}\left(x_{2}\right)=6 ; k_{\Delta}=\beta / N_{0}=0.05$} \\
\hline$\mu[\mathrm{dB}]$ & $\begin{array}{l}\text { Nonconstrained RSF } \\
\text { IOSNR }^{(2)}\end{array}$ & $\begin{array}{l}\text { Constrained RSF } \\
\text { IOSNR }^{(3)}\end{array}$ & $\begin{array}{l}\text { Nonconstrained RASF } \\
\operatorname{IOSNR}^{(4)}\end{array}$ & $\begin{array}{c}\text { Constrained RASF (WCSP-optimized) } \\
\operatorname{IOSNR}^{(5)}\end{array}$ \\
\hline 5 & 1.71 & 2.17 & 1.9 & 2.41 \\
\hline 10 & 1.85 & 2.61 & 1.92 & 2.88 \\
\hline 15 & 1.9 & 2.9 & 2.2 & 3.45 \\
\hline 20 & 1.93 & 3.4 & 2.18 & 4.16 \\
\hline 25 & 2.01 & 3.78 & 2.6 & 4.56 \\
\hline 30 & 2.11 & 4.3 & 3.08 & 5.32 \\
\hline
\end{tabular}

inverse of the signal-to-noise ratio (SNR), that is, $\alpha=\left(N_{0}+\right.$ $\beta) / b_{0}$, where $b_{0}$ is the prior average gray level of the SSP, and the uncertainty factor $\beta$ is attributed to $\alpha$. In that case, the SO $\mathrm{F}$ is recognized to be the Tikhonov-type robust spatial filter (RSF):

$$
\mathbf{F}_{\mathrm{RSF}}=\left(\mathbf{S}^{+} \mathbf{S}+\left(\left(N_{0}+\beta\right) / b_{0}\right) \mathbf{I}\right)^{-1} \mathbf{S}^{+}
$$

\subsubsection{Matched spatial filtering algorithm}

Consider the model from the previous example for an assumption, $\alpha \gg\left\|S^{+} \mathbf{S}\right\|$, that is, the case of a priority of the second error measure (suppression of noise) over the systematic error in the optimization problem (17). In this case, we can roughly approximate (20), (24) as the matched spatial filter (MSF):

$$
\mathrm{F}_{\mathrm{MSF}} \approx \text { const. } \mathrm{S}^{+}
$$

where the normalizing constant is irrelevant as it specifies the constant image scaling factor that does not influence the overall reconstructed image pattern.

\subsubsection{Robust adaptive spatial filtering algorithm}

Consider the case of an arbitrary zero-mean noise with the composed correlation matrix $\mathbf{R}_{\Sigma}$, equal importance of two error measures in (18), that is, $\alpha=1$, and the solutiondependent weight matrix $\mathbf{A}=\hat{\mathbf{D}}=\operatorname{diag}\{\hat{\mathbf{b}}\}$. In this case, the
SO becomes the robust adaptive (i.e., solution-dependent) spatial filter (RASF) operator:

$$
\mathbf{F}_{\mathrm{RASF}}=\left(\mathbf{S}^{+} \mathbf{R}_{\Sigma}^{-1} \mathbf{S}+\hat{\mathbf{D}}^{-1}\right)^{-1} \mathbf{S}^{+} \mathbf{R}_{\Sigma}^{-1}
$$

The three SSP reconstruction techniques that employ the SOs (24), (25), and (26) compose the family of the DEDRrelated estimators:

$$
\hat{b}_{(K)}^{(p)}(\mathbf{x})=\mathbf{g}^{T}(\mathbf{x}) \operatorname{diag}\left\{\left\{\mathbf{F}^{(p)} \mathbf{Y} \mathbf{F}^{(p)+}\right\}_{\operatorname{diag}}\right\} \mathbf{g}(\mathbf{x}), \quad p=1,2,3
$$

with $\mathbf{F}^{(1)}=\mathbf{F}_{\mathrm{MSF}}, \mathbf{F}^{(2)}=\mathbf{F}_{\mathrm{RSF}}$, and $\mathbf{F}^{(3)}=\mathbf{F}_{\mathrm{RASF}}$, respectively. Any other feasible adjustments of the DEDR degrees of freedom (the regularization parameters $\alpha, \beta$, and the weight matrix A) provide other possible DEDR-related SSP reconstruction techniques numbered further on as $p=4, \ldots$. As an important example, in the sequential subsection, we show that such DEDR family encompasses also the celebrated minimum variance distortionless response (MVDR) beamforming method transformed into the high-resolution RSS estimation technique with the proper MVDR SO $\mathbf{F}_{\mathrm{MVDR}}=$ $\mathbf{F}^{(4)}$ specified further on by (31).

\subsection{Relationship with the robust MVDR beamformer}

The conventional MVDR beamformer [7] "reconstructs" the RS image by minimizing the power or variance of the adaptive array output for all search directions, $k=1, \ldots, K$, under the constraint that the gain in the particular look 


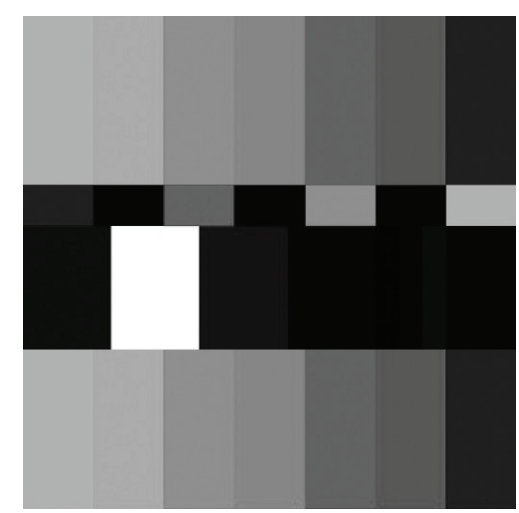

(a)

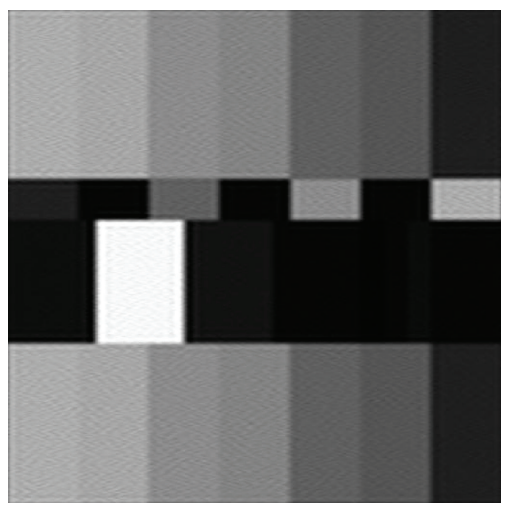

(c)

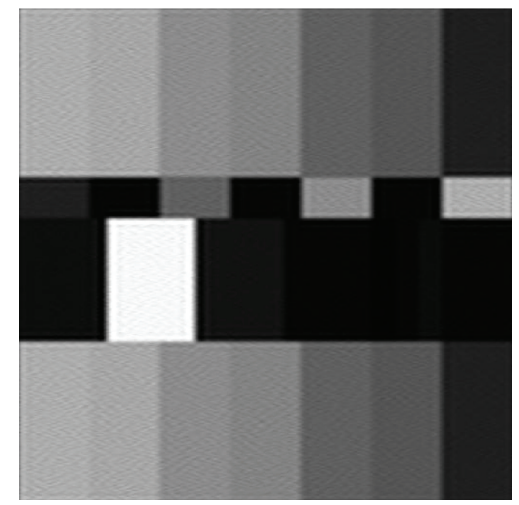

(e)

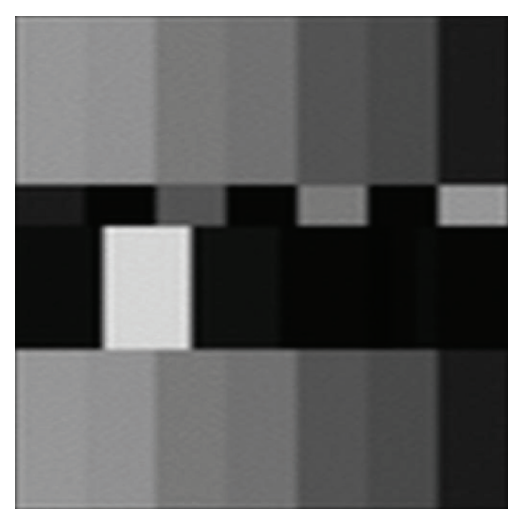

(b)

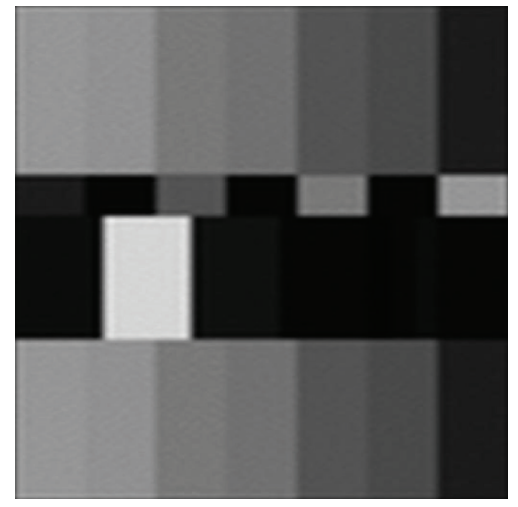

(d)

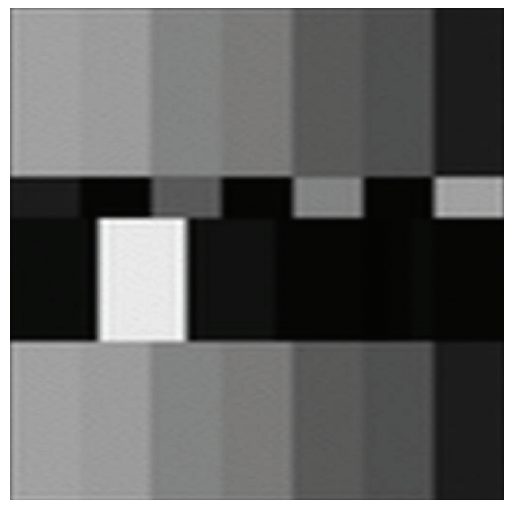

(f)

FIGURE 1: First operational scenario, first scene $(\mu=20 \mathrm{~dB})$ : (a) artificially synthesized original scene; (b) degraded uncertain scene image formed applying the MSF method; (c) image reconstructed applying the nonconstrained RSF algorithm; (d) image reconstructed with the constrained RSF algorithm; (e) image reconstructed applying the nonconstrained RASF algorithm; and (f) image reconstructed applying the constrained RASF (WCSP-optimized) algorithm.

direction is equal to a constant (one, for simplicity). This results in the well-known conventional MVDR algorithm $[7,10]$ :

$$
\hat{b}_{k}=\left(\mathbf{s}_{k}^{+} \mathbf{Y}^{-1} \mathbf{s}_{k}\right)^{-1} ; \quad k=1, \ldots, K,
$$

where $\boldsymbol{s}_{k}$ represents the so-called "steering vector" for the $k$ th look direction, which in our notations is essentially the $k$ th column vector of the nominal SFO matrix $\mathbf{S}$.
For the purposes of establishing a relationship between the MVDR beamformer and the DEDR-related SSP estimators (27), we now rewrite the conventional MVDR algorithm (28) as

$$
\hat{\mathbf{b}}_{\text {MVDR }}=\left\{\left[\operatorname{diag}\left\{\left\{\mathbf{S}^{+} \mathbf{Y}^{-1} \mathbf{S}\right\}_{\text {diag }}\right\}\right]^{-1}\right\}_{\text {diag }}
$$

that can be considered as a solution to the equation, $\hat{\mathbf{D}}=$ $\widehat{\mathbf{D}} \mathbf{S}^{+} \mathbf{Y}^{-1} \mathbf{S} \hat{\mathbf{D}}$. Expressing now $\mathbf{Y}^{-1}=\mathbf{Y}^{-1} \mathbf{Y Y}^{-1}$ and using the 


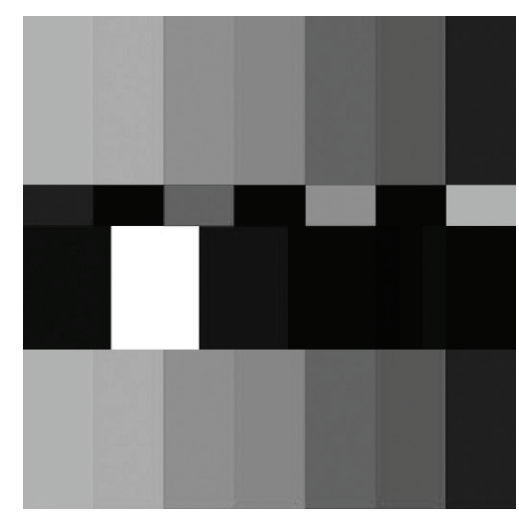

(a)

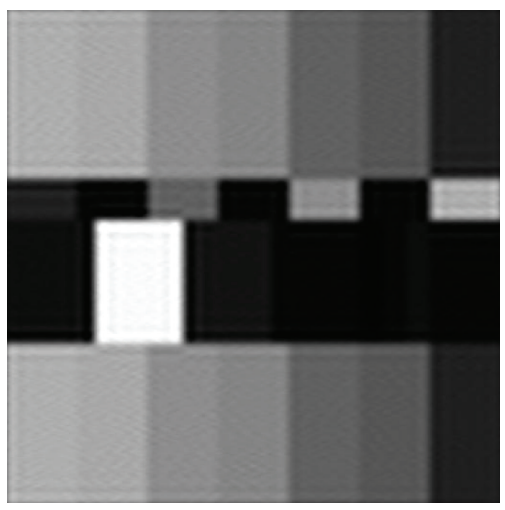

(c)

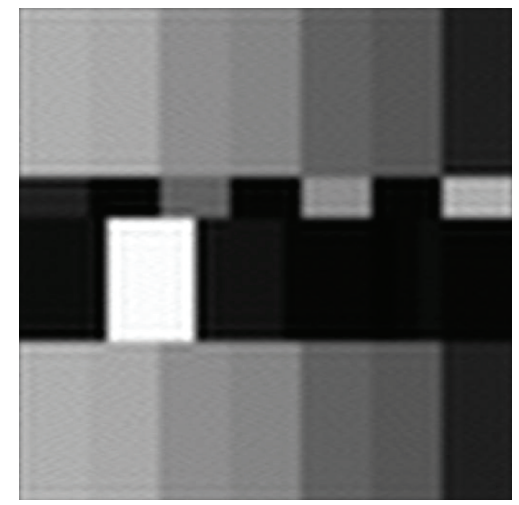

(e)

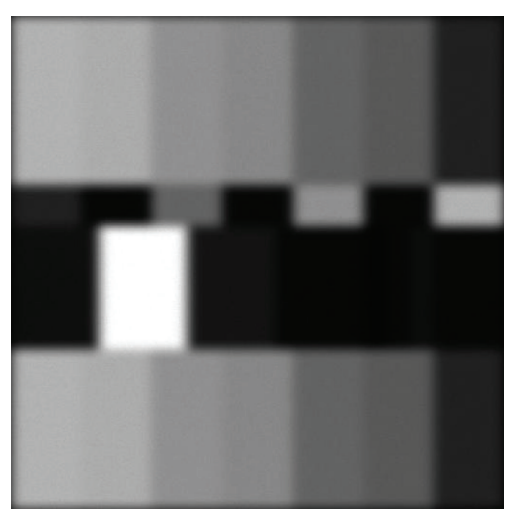

(b)

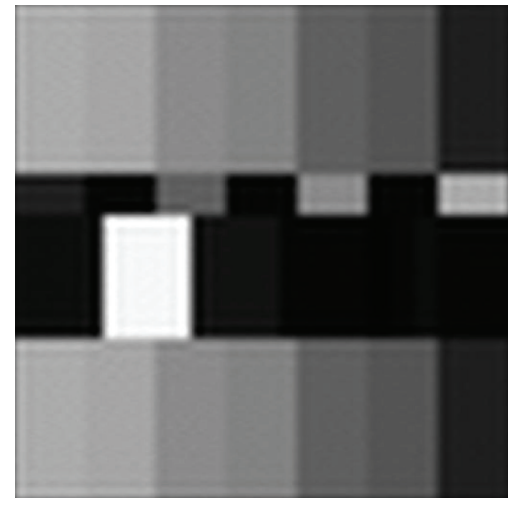

(d)

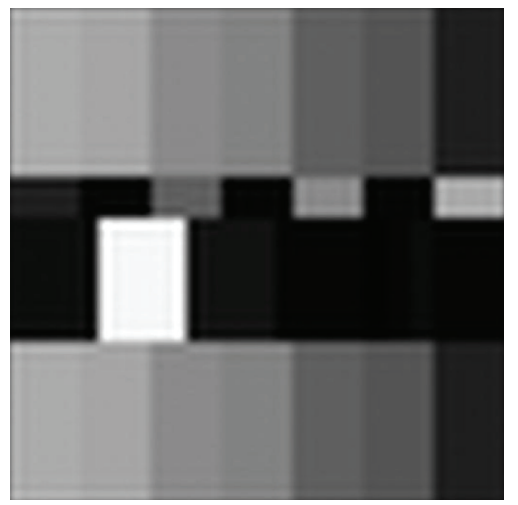

(f)

FIgURE 2: Second operational scenario, first scene $(\mu=20 \mathrm{~dB}$ ): (a) artificially synthesized original scene; (b) degraded uncertain scene image formed applying the MSF method; (c) image reconstructed applying the nonconstrained RSF algorithm; (d) image reconstructed with the constrained RSF algorithm; (e) image reconstructed applying the nonconstrained RASF algorithm; and (f) image reconstructed applying the constrained RASF (WCSP-optimized) algorithm.

second-form representation [15] for the operator, $\hat{\mathbf{D}} \mathbf{S}^{+} \mathbf{Y}^{-1}=$ $\left(\mathbf{S}^{+} \mathbf{R}_{\mathbf{n}}^{-1} \mathbf{S}+\hat{\mathbf{D}}^{-1}\right)^{-1} \mathbf{S}^{+} \mathbf{R}_{\mathbf{n}}^{-1}$, we obtain the alternative representation form for the MVDR algorithm (28), that is,

$$
\hat{\mathbf{b}}_{\mathrm{MVDR}}=\left\{\mathbf{F}_{\mathrm{MVDR}} \mathbf{Y F _ { M V D R } ^ { + }}\right\}_{\text {diag }}
$$

with

$$
\mathbf{F}_{\mathrm{MVDR}}=\mathbf{F}^{(4)}=\hat{\mathbf{D}} \mathbf{S}^{+} \mathbf{Y}^{-1}=\left(\mathbf{S}^{+} \mathbf{R}_{\mathbf{n}}^{-1} \mathbf{S}+\widehat{\mathbf{D}}^{-1}\right)^{-1} \mathbf{S}^{+} \mathbf{R}_{\mathbf{n}}^{-1} .
$$

Examining the formulae (20), (21), and (31), one may deduce that $\mathbf{F}_{\text {MVDR }}=\mathbf{F}^{(4)}$ coincides with $\mathbf{F}_{\text {DEDR }}$ for the nonrobust adaptive case, that is, $\beta=0, \mathbf{A}=\hat{\mathbf{D}}$.

\section{SIMULATIONS AND DISCUSSIONS}

We simulated a conventional side-looking SAR with the fractionally synthesized aperture, that is, the array was synthesized by the moving antenna. The regular SFO of such 
SAR is factored along two axes in the image plane [17, 18]: the azimuth or cross-range coordinate (horizontal axis, $x_{1}$ ) and the slant range (vertical axis, $x_{2}$ ). In the simulations, we considered the conventional triangular SAR range ambiguity function (AF) $\Psi_{r}\left(x_{2}\right)$ and two approximations of the SAR azimuth AF: (i) "sinc" approximation, $\Psi_{a}^{(1)}\left(x_{1}\right)=\left|\operatorname{sinc}\left(x_{1} / a\right)\right|$, and (ii) Gaussian "bell" approximation, $\Psi_{a}^{(2)}\left(x_{1}\right)=\exp \left(-\left(x_{1}\right)^{2} / a^{2}\right)$, with the adjustable fractional parameters $a[15]$. Note that in the imaging radar theory $[3,8]$, the AF is referred to as the continuous-form approximation of the ambiguity operator matrix $\Psi=S^{+} \mathbf{S}$ and serves as an equivalent to the point spread function in the conventional image processing terminology $[16,19]$. In this paper, we present the simulations performed with two characteristic scenes. The fist one of the 512-by-512 pixel format was artificially generated. The second one of the same 512-by-512 pixel format was borrowed from the real world high-resolution terrain SAR imagery (south-west Guadalajara region, Mexico [20]). The first scene was used as a test for adjustment of the degrees of freedom of the developed RSF and RASF algorithms to attain the desired improvement in the image enhancement performances (the IOSNR defined below). In the reported simulations, the representation formats along the $x_{2}$ (slant range) and $x_{1}$ (cross range, i.e., azimuth) directions were adjusted to the same effective pixel width. In the $x_{1}$ direction, the fractional parameter $a$ was controlled to adjust different effective widths $\Delta \Psi_{a}\left(x_{1}\right)$ of the azimuth AF. The corresponding adjustment of different effective width of the range AF $\Delta \Psi_{r}\left(x_{2}\right)$ was performed over the slant range direction $\left(x_{2}\right)$.

For the purpose of objectively testing the performances of different DEDR-related SSP estimation algorithms, a quantitative evaluation of the improvement in the SSP estimates (gained due to applying the DEDR-related reconstructive solution operators $\mathbf{F}^{(p)} ; p=2, \ldots$, instead of the MSF, i.e., the adjoint operator $\mathbf{F}^{(1)}=\mathbf{S}^{+}$) was accomplished. In analogy to image reconstruction quality metrics $[16,19]$, we adopt here the quality metric defined as an improvement in the output signal-to-noise ratio (IOSNR):

$$
\operatorname{IOSNR}^{(p)}=10 \log _{10} \frac{\sum_{k=1}^{K}\left(\hat{b}_{k}^{(\mathrm{MSF})}-b_{k}\right)^{2}}{\sum_{k=1}^{K}\left(\hat{b}_{k}^{(p)}-b_{k}\right)^{2}}, \quad p=2,3,4,5,
$$

where $b_{k}$ represents a value of the $k$ th element (pixel) of the original SSP $\mathbf{b}, \hat{b}_{k}^{(\mathrm{MSF})}$ represents a pixel value of the $k$ th element (pixel) of the rough SSP estimate $\hat{\mathbf{b}}_{\mathrm{MSF}}$ formed applying the matched spatial filtering technique (conventional matched beamformer with $\mathbf{F}^{(1)}=\mathbf{S}^{+}$), and $\hat{b}_{k}^{(p)}$ represents a value of the $k$ th pixel of the SSP reconstructed from the matched $\hat{\mathbf{b}}_{\mathrm{MSF}}$ applying one of the particular developed DEDR-related SOs. In the simulation studies, four different DEDR-related estimators were tested, renumbered here as $p=2,3,4$, and 5 . The $\mathbf{F}^{(2)}$ corresponds to the nonconstrained $\mathbf{F}_{\mathrm{RSF}}$, that is, to the RSF method adjusted incorrectly to the scenario assuming no uncertainties in the data $(\beta=0)$. The $\mathbf{F}^{(3)}$ corresponds to the constrained $\mathbf{F}_{\mathrm{RSF}}$ with the SFO uncertainty factor $k_{\Delta}=\beta / N_{0}$ correctly adjusted to two different uncertain scenarios (as specified in Tables 1 and 2). The $\mathbf{F}^{(4)}$ corresponds to the nonconstrained RASF, that is, the RASF method adjusted incorrectly to the scenario with no uncertainties in the data $(\beta=0)$. Last, the $\mathbf{F}^{(5)}$ corresponds to the constrained $\mathbf{F}_{\mathrm{RASF}}$ with the SFO uncertainty factor $k_{\Delta}=\beta / N_{0}$ correctly adjusted to two different uncertain scenarios (as specified in Tables 1 and 2), that is, the WCSP-optimized DEDR estimator. According to the quality metric (32), the higher the IOSNR, the better the improvement in the SSP estimate is, that is, the closer the estimate is to the original SSP.

In this section, we report the qualitative simulation results and the relevant quantitative performances evaluated via the IOSNRs (32) (in the $\mathrm{dB}$ scale) gained with these four robust DEDR-related estimators, in particular: $\operatorname{IOSNR}^{(2)}$ gained using the nonconstrained RSF in the uncertain scenario; $\mathrm{IOSNR}^{(3)}$ gained applying the constrained RSF in the same uncertain scenario; $\operatorname{IOSNR}^{(4)}$ gained using the nonconstrained RASF; and $\operatorname{IOSNR}^{(5)}$ gained applying the constrained RASF (WCSP-optimized estimator) in the same uncertain scenario. The simulation experiments were run for two typical SAR systems that operate under different SNRs levels $\mu=b_{0} / N_{0}$, different fractionally synthesized apertures (characterized by the width of the azimuth AFs $\Delta \Psi_{a}\left(x_{1}\right)$ ), and different uncertainty factors $k_{\Delta}=\beta / N_{0}$ (as specified in Tables 1 and 2 ) that bound via (11), (19) the impact of the uncertainty SFO term. In particular, the simulated scenarios are specified as follows.

(i) First uncertain operational scenario (simulation experiment specifications):

(a) fractional azimuth AF width, $\Delta \Psi_{a}^{(1)}\left(x_{1}\right)=10$ pixels of the $512 \times 512$ scene pixel format (at the 0.5 from the peak value of the "sinc-type" $\left.\operatorname{AF}, \Psi_{a}^{(1)}\left(x_{1}\right)=\left|\operatorname{sinc}\left(x_{1} / a\right)\right|\right)$;

(b) range AF width, $\Delta \Psi_{r}\left(x_{2}\right)=3$ pixels (at the 0.5 from the peak value of the triangular $\left.\Psi_{r}\left(x_{2}\right)\right)$;

(c) SNRs range, $\mu=b_{0} / N_{0}=5 \mathrm{~dB}, \ldots, 30 \mathrm{~dB}$;

(d) SFO uncertainty factor, $k_{\Delta}=\beta / N_{0}=0.1$.

(ii) Second uncertain operational scenario (simulation experiment specifications):

(a) fractional azimuth AF width, $\Delta \Psi_{a}^{(2)}\left(x_{1}\right)=14$ pixels (at the 0.5 from the peak value of the "bell-type" $\left.\mathrm{AF}, \Psi_{a}^{(2)}\left(x_{1}\right)=\exp \left(-\left(x_{1}\right)^{2} / a^{2}\right)\right)$;

(b) range AF width, $\Delta \Psi_{r}\left(x_{2}\right)=6$ pixels (at the 0.5 from the peak value of the triangular $\left.\Psi_{r}\left(x_{2}\right)\right)$;

(c) SNRs range, $\mu=b_{0} / N_{0}=5 \mathrm{~dB}, \ldots, 30 \mathrm{~dB}$;

(d) SFO uncertainty factor, $k_{\Delta}=\beta / N_{0}=0.05$.

These specifications correspond to two typical uncertain scenarios with airborne SAR sensor trajectory deviations modelled in [17].

Figures 1(a) and 2(a) show the same artificially synthesized test scene. Figures 3(a) and 4(a) show the second tested original scene (borrowed from the real world high-resolution 


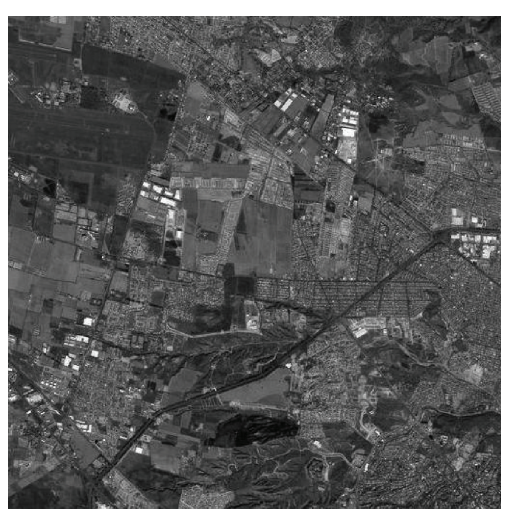

(a)

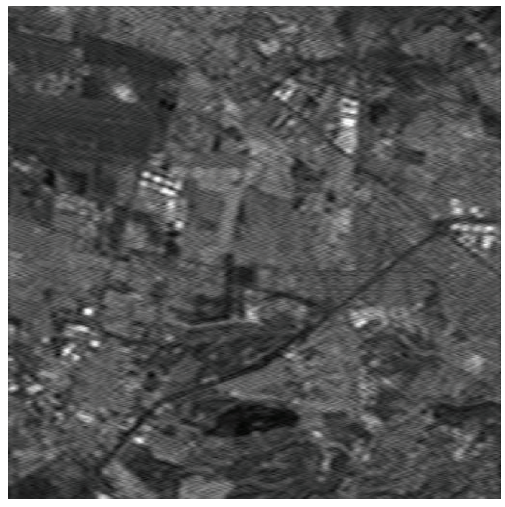

(c)

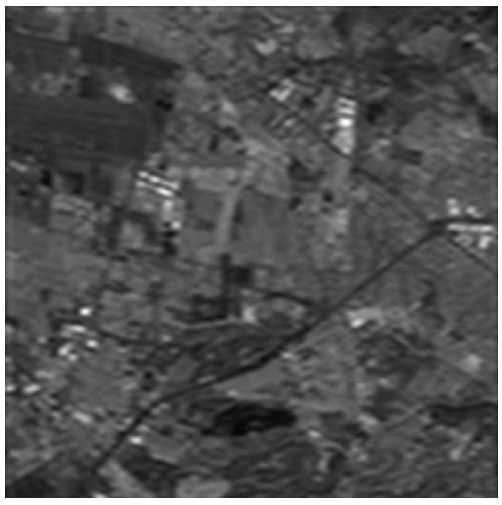

(e)

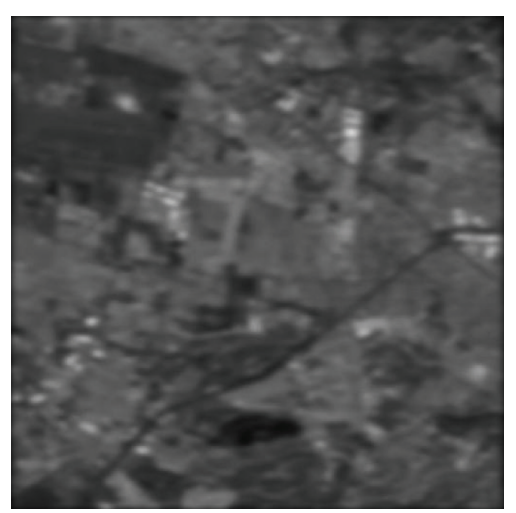

(b)

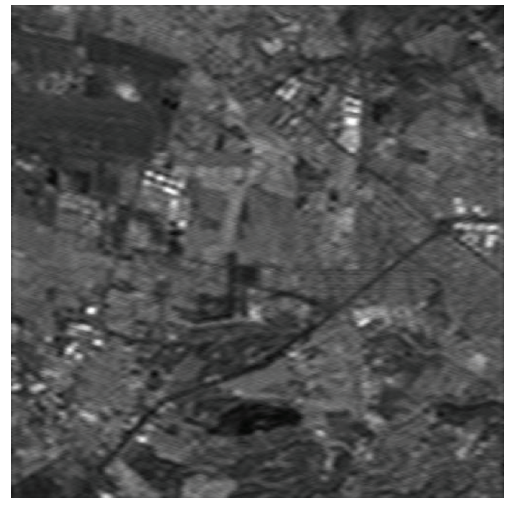

(d)

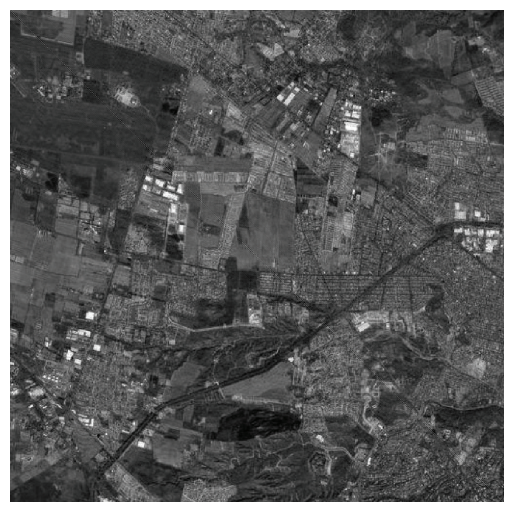

(f)

FIGURE 3: First operational scenario, second scene $(\mu=20 \mathrm{~dB})$ : (a) original scene; (b) degraded uncertain scene image formed applying the MSF method; (c) image reconstructed applying the nonconstrained RSF algorithm; (d) image reconstructed with the constrained RSF algorithm; (e) image reconstructed applying the nonconstrained RASF algorithm; and (f) image reconstructed applying the constrained RASF (WCSP-optimized) algorithm.

SAR imagery [20]). The remaining images of Figure 1 through Figure 4 present the results of image formation applying different DEDR-related SSP estimators as specified in the figure captions. Figures 1(b) through 4(b) demonstrate the images formed applying the conventional MSF for the uncertain fractionally synthesized SAR scenarios. According to the EO (6), the overall uncertain data degradations $\tilde{\mathbf{n}}=\Delta \mathbf{e}+\mathbf{n}$ were composed of a mixture of conventional white additive observation noise $\mathbf{n}$ and correlated (scenedependent) multiplicative noise $\Delta \mathbf{e}$. Following the DEDR methodology (detailed in Section 3), the SFO uncertainty cannot be factorized into separate terms caused by the environmental perturbations, SAR trajectory deviations, or antenna vibrations. Thus, the composed multiplicative degradation effect was modeled via simulating the MSF scene image corrupted by the speckle noise via incorporating into (9) with the SO (25), the uncertain operational scenario factors, in particular, the uncertain data model correlation matrix $\mathbf{Y}$ that corresponds to the degraded EO (6) with the diagonal loaded noise augmented correlation matrix (19). Figures 1(c) through 4(c) show the enhanced images formed applying the unconstrained RSF, that is, the RSF 


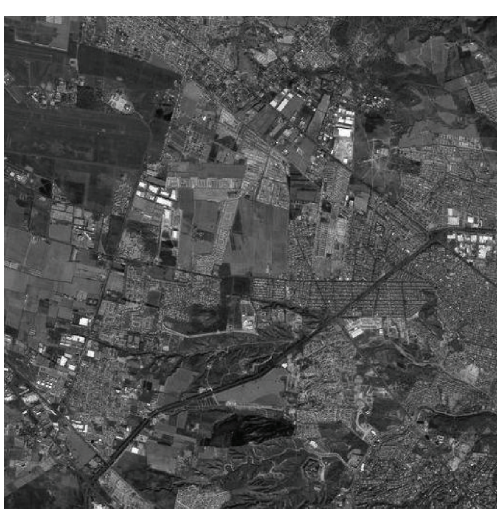

(a)

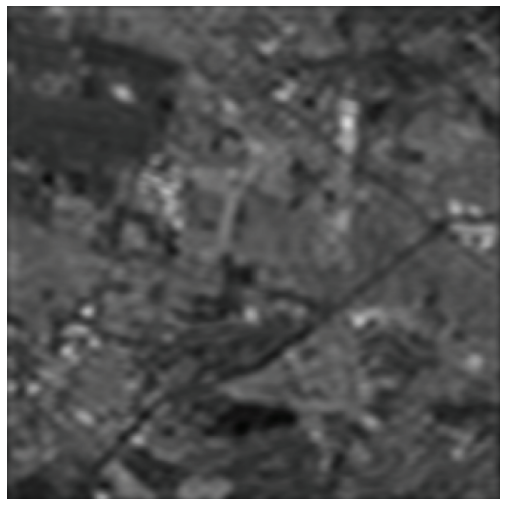

(c)

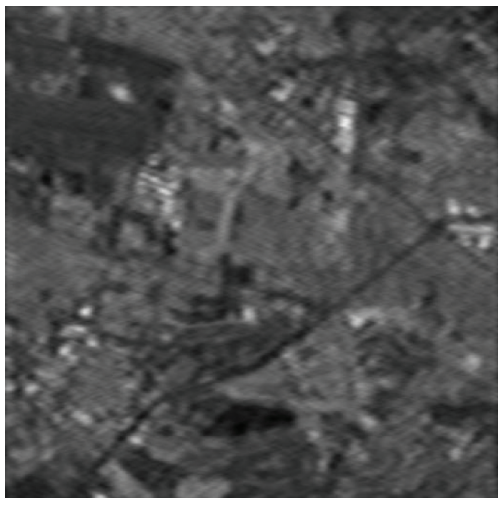

(e)

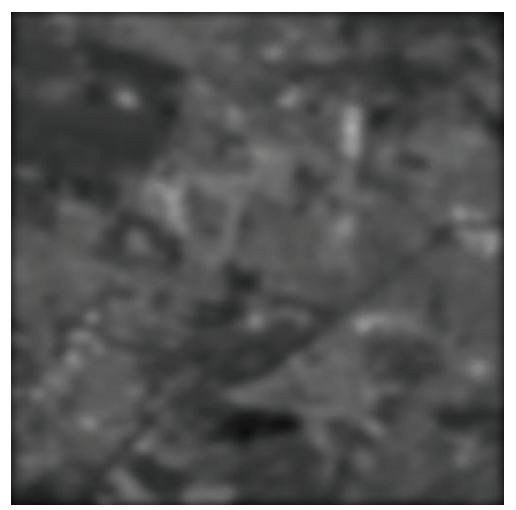

(b)

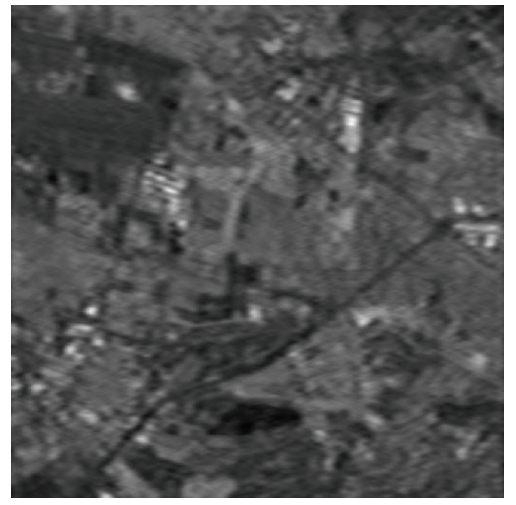

(d)

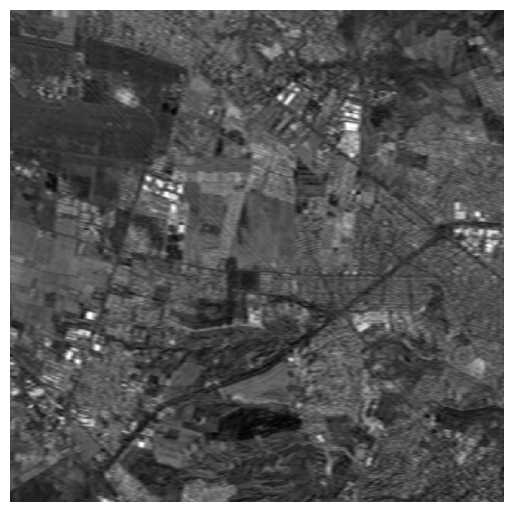

(f)

FIGURE 4: Second operational scenario, second scene $(\mu=20 \mathrm{~dB}$ ): (a) original scene; (b) degraded uncertain scene image formed applying the MSF method; (c) image reconstructed applying the nonconstrained RSF algorithm; (d) image reconstructed with the constrained RSF algorithm; (e) image reconstructed applying the nonconstrained RASF algorithm; and (f) image reconstructed applying the constrained RASF (WCSP-optimized) algorithm.

incorrectly adjusted to the uncertain scenario via ignoring the uncertainty factor $(\beta=0)$. Figures $1(d)$ through $4(d)$ present the enhanced images formed using the constrained RSF properly adjusted to the particular uncertain scenario $\left(k_{\Delta}=\beta / N_{0}=0.1\right.$ for the first scenario, and $k_{\Delta}=\beta / N_{0}$ $=0.05$ for the second scenario, respectively). The images enhanced with the unconstrained RASF $(\beta=0)$ are shown in Figures $1(\mathrm{e})-4(\mathrm{e})$, and the corresponding images reconstructed with the constrained RASF (WCSP-optimized method) are presented in Figures 1(f) -4(f), respectively.
From the presented simulation results, the advantage of the well-designed imaging experiments (constrained RSF and WCSP-optimized RASF) over the case of badly designed experiment (nonrobust MSF and unconstrained RSF) is evident. Due to the performed regularized inversions, the resolution was substantially improved in all simulated scenarios (as reported in Tables 1 and 2). The higher values of $\operatorname{IOSNR}^{(3)}>\operatorname{IOSNR}^{(2)}$ as well as $\operatorname{IOSNR}^{(5)}>\operatorname{IOSNR}^{(4)}$ were obtained with the constrained DEDR-related estimators, that is, with the DEDR techniques adopted to the uncertain 
scenarios. Note that IOSNR (32) is basically a square-type error metric. Thus, it does not qualify quantitatively the "delicate" visual features in the reconstructed images; hence, small differences in the corresponding IOSNRs reported in Tables 1 and 2. In addition, both enhanced robust estimators manifest the higher IOSNRs in the case of more smooth azimuth AFs (larger $\left.\Delta \Psi_{a}\left(x_{1}\right)\right)$ and higher SNRs $\mu$. For the DEDR-optimized RASF method, in addition, the ringing (image speckle) effect was substantially reduced, while the nonadaptive constrained RSF estimator requires considerably less computational load. These results qualitatively demonstrate that with proper adjustment of the degrees of freedom in the developed DEDR estimators (24), (27), one could approach the quality of the DEDR-optimal image formation method (22) avoiding the cumbersome adaptive computations required to implement the DEDR-optimal algorithm [10, 15].

\section{CONCLUSION}

New descriptive experiment design regularization (DEDR) approach for estimation of the spatial spectrum pattern (SSP) of the wavefield power distribution in the uncertain remotely sensed environment has been proposed as required for the conventional array imaging radar, side-looking airborne radar, and SAR. Unifying the DEDR and the worstcase statistical performance (WCSP) optimization into the aggregated WCSP-constrained minimum risk technique, the inverse problem ill-posedness has been alleviated in a statistically grounded fashion. The derived general-form DEDR estimator does not involve the inversion of the estimated data correlation matrix. This principal algorithmic-level result of the undertaken study constitutes the crucial advantage of the developed family of the DEDR-related estimators that makes them applicable to the uncertain operational scenarios with ill-conditioned (e.g., low-rank) estimates of the array data correlation matrices, in particular, to the SAR imaging scenarios where only one realization of the trajectory data signal degraded due to the uncontrolled random carrier trajectory deviation and antenna vibration is available for further processing. Being nonlinear and solution-dependent, the DEDR-optimal robust adaptive spatial filtering (RASF) estimator requires rather complex signal processing. The computational complexity arises due to the necessity to perform simultaneously the solution-dependent operator inversion operations and adaptive adjustments of the degrees of freedom of the overall RASF technique. To reduce the computational load, the simplified constrained robust spatial filtering (RSF) algorithm was proposed and employed, which manifests almost the same reconstruction performances as the RASF in typical uncertain operational scenarios that was verified in the simulation experiment.

\section{REFERENCES}

[1] S. E. Falkovich, V. I. Ponomaryov, and Y. Shkvarko, Optimal Reception of Space-Time Signals in Channels with Scattering, Radio i Sviaz,Moscow, Russia, 1989.
[2] G. T. Capraro, A. Farina, H. Griffiths, and M. C. Wicks, "Knowledge-based radar signal and data processing: a tutorial review," IEEE Signal Processing Magazine, vol. 23, no. 1, pp. 18-29, 2006.

[3] D. R. Wehner, High-Resolution Radar, Artech House, Boston, Mass, USA, 2nd edition, 1994.

[4] H. L. Van Trees, Detection, Estimation and Modulation Theory, Part IV, Optimum Array Processing, Wiley-Interscience, New York, NY, USA, 2002.

[5] S. M. Kay, Modern Spectral Estimation, Prentice-Hall, Englewood Cliffs, NJ, USA, 1988.

[6] S. Haykin and A. Steinhardt, Eds., Adaptive Radar Detection and Estimation, John Willey \& Sons, New York, NY, USA, 1992.

[7] P. Stoica and R. L. Moses, Introduction to Spectral Analysis, Prentice-Hall, Englewood Cliffs, NJ, USA, 1997.

[8] F. M. Henderson and A. V. Lewis, Eds., Principles and Applications of Imaging Radar, vol. 2 of Manual of Remote Sensing, John Willey \& Sons, New York, NY, USA, 3rd edition, 1998.

[9] S. A. Vorobyov, A. B. Gershman, and Z.-Q. Luo, "Robust adaptive beamforming using worst-case performance optimization: a solution to the signal mismatch problem," IEEE Transactions on Signal Processing, vol. 51, no. 2, pp. 313-324, 2003.

[10] A. B. Gershman, "Robustness issues in adaptive beamforming and high-resolution direction finding," in High-Resolution and Robust Signal Processing, Y. Hua, A. B. Gershman, and Q. Cheng, Eds., pp. 63-100, Marcel Dekker, New York, NY, USA, 2004.

[11] D. W. Rieken and D. R. Fuhrmann, "Generalizing MUSIC and MVDR for multiple noncoherent arrays," IEEE Transactions on Signal Processing, vol. 52, no. 9, pp. 2396-2406, 2004.

[12] J. Li, P. Stoica, and Z. Wang, "Doubly constrained robust Capon beamformer," IEEE Transactions on Signal Processing, vol. 52, no. 9, pp. 2407-2423, 2004.

[13] Y. Shkvarko, "Estimation of wavefield power distribution in the remotely sensed environment: Bayesian maximum entropy approach," IEEE Transactions on Signal Processing, vol. 50, no. 9, pp. 2333-2346, 2002.

[14] Y. Shkvarko, "Unifying regularization and Bayesian estimation methods for enhanced imaging with remotely sensed datapart II: implementation and performance issues," IEEE Transactions on Geoscience and Remote Sensing, vol. 42, no. 5, pp. 932-940, 2004.

[15] Y. Shkvarko, "From matched spatial filtering towards the fused statistical descriptive regularization method for enhanced radar imaging," EURASIP Journal on Applied Signal Processing, vol. 2006, Article ID 39657, 9 pages, 2006.

[16] H. H. Barrett and K. J. Myers, Foundations of Image Science, Wiley-Interscience, New York, NY, USA, 2004.

[17] G. Franceschetti, A. Iodice, S. Perna, and D. Riccio, "SAR sensor trajectory deviations: Fourier domain formulation and extended scene simulation of raw signal," IEEE Transactions on Geoscience and Remote Sensing, vol. 44, no. 9, pp. 2323-2334, 2006.

[18] M. S. Greco and F. Gini, "Statistical analysis of high-resolution SAR ground clutter data," IEEE Transactions on Geoscience and Remote Sensing, vol. 45, no. 3, pp. 566-575, 2007.

[19] V. Ponomaryov, A. Rosales, F. Gallegos, and I. Loboda, "Adaptive vector directional filters to process multichannel images," IEICE Transactions on Communications, vol. E90-B, no. 2, pp. 429-430, 2007.

[20] Space Imaging, GeoEye Inc., 2007, http://www.geoeye.com/. 

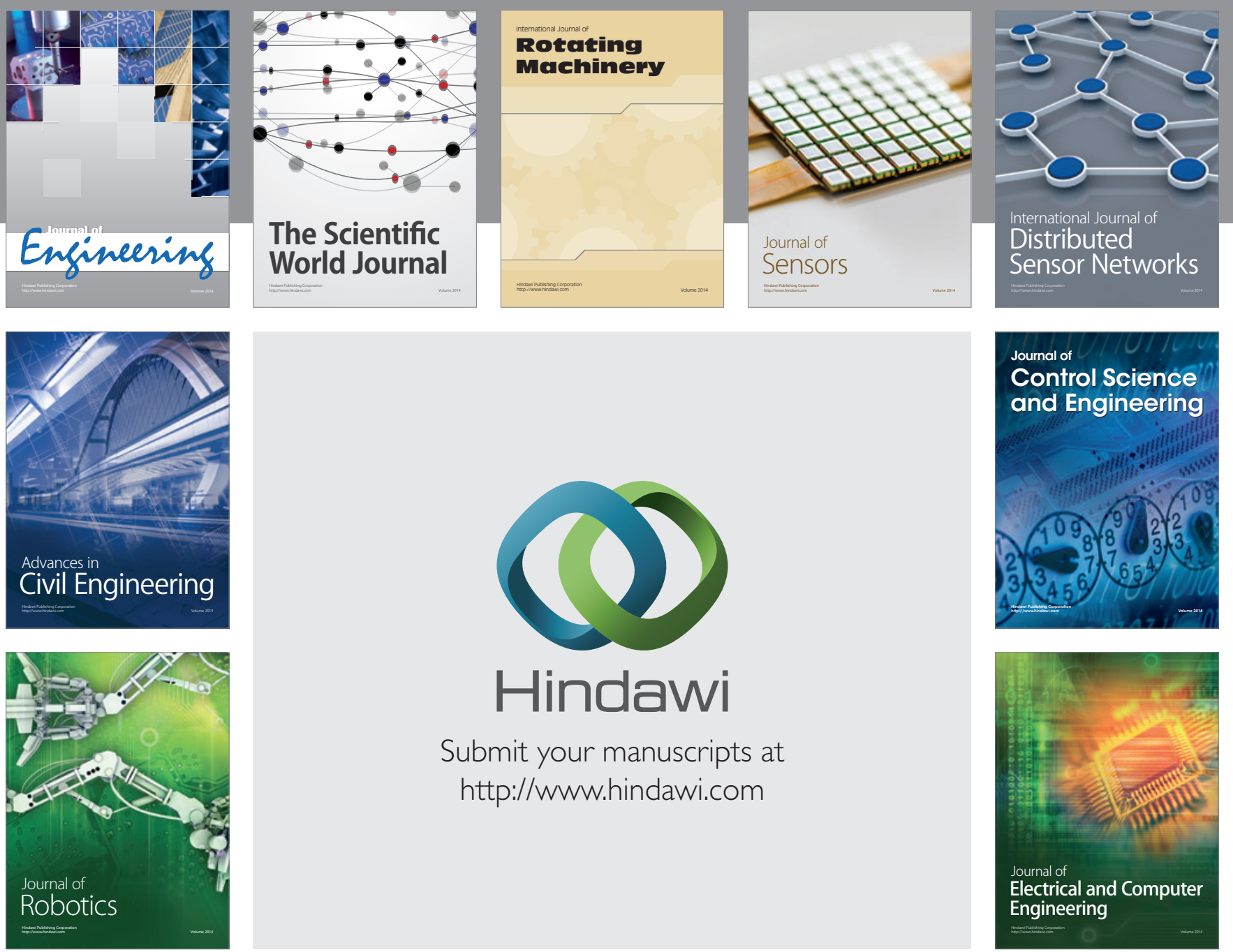

Submit your manuscripts at

http://www.hindawi.com
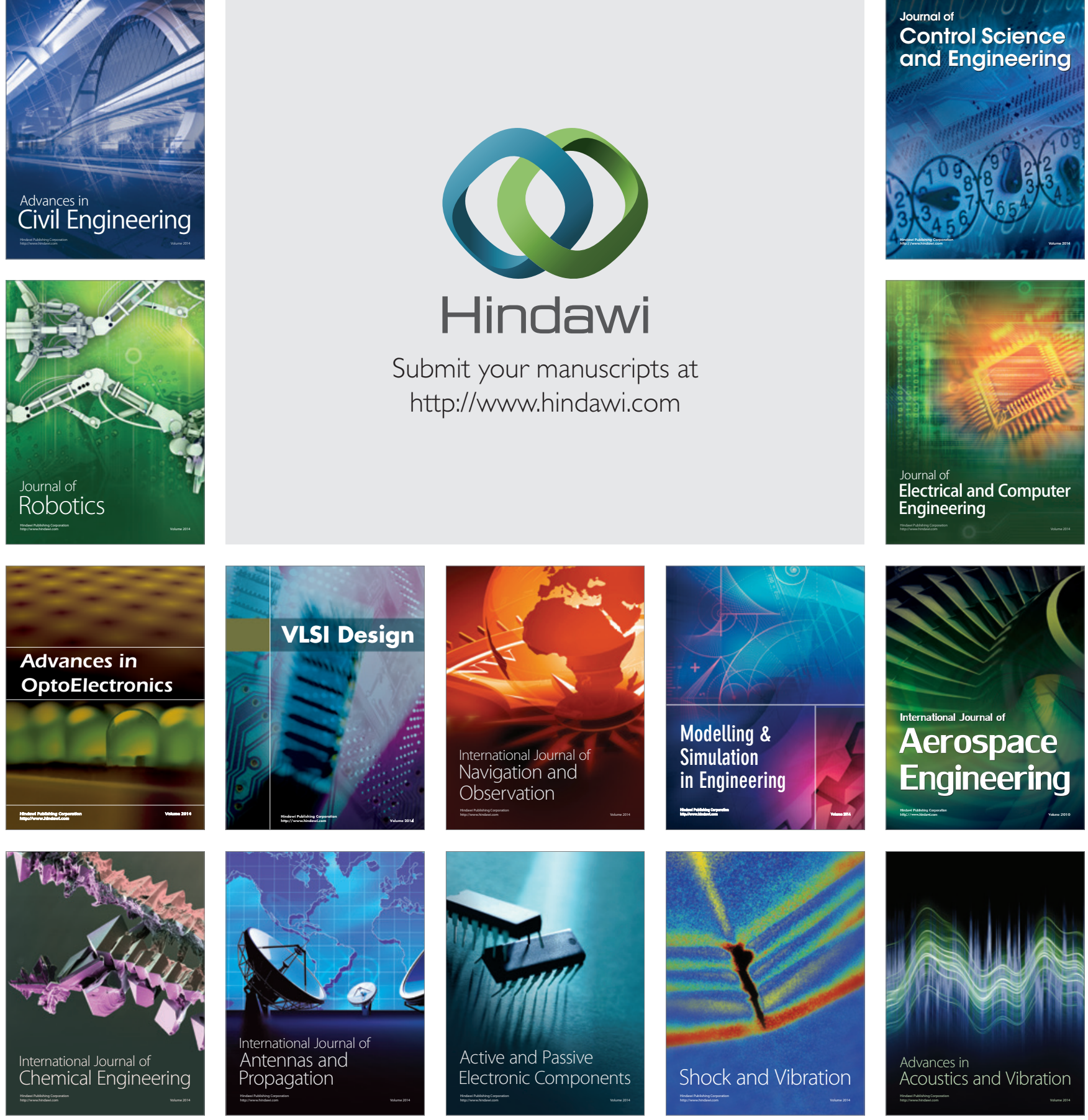